Research Article

\title{
Prediction of Ionospheric TEC Based on the NARX Neural Network
}

\author{
Liu Guoyan $\mathbb{D}^{1},{ }^{1}$ Gao Wang, ${ }^{2}$ Zhang Zhengxie, ${ }^{2}$ and Zhao Qing ${ }^{2}$ \\ ${ }^{1}$ Nanjing Vocational University of Industry Technology, Nanjing 210000, China \\ ${ }^{2}$ South and East University, Nanjing 210000, China \\ Correspondence should be addressed to Liu Guoyan; 2296813027@qq.com
}

Received 27 August 2021; Revised 1 October 2021; Accepted 15 October 2021; Published 31 October 2021

Academic Editor: Chao $\mathrm{Hu}$

Copyright (c) 2021 Liu Guoyan et al. This is an open access article distributed under the Creative Commons Attribution License, which permits unrestricted use, distribution, and reproduction in any medium, provided the original work is properly cited.

Effective prediction of ionospheric total electron content (TEC) is very important for Global Navigation Satellite System (GNSS) positioning and other related applications. This paper proposes an ionospheric TEC prediction method using the nonlinear autoregressive with exogenous input (NARX) neural network, which uses previous TEC data and external time parameter inputs to establish a TEC prediction model. During the years of different solar activities, 12 datasets of 3 stations with different latitudes are used for experiments. Each dataset uses the first 120 days for training and the next 20 days for testing. For each test dataset, a sliding window strategy is adopted in the prediction process, wherein the TEC of future 2 days are predicted by the true TEC values of the previous 2 days. The results show that in the year with active solar activity (2011), the TEC prediction with the NARX network can improve the accuracy by $32.3 \%$ and $43.5 \%$, compared with the autoregressive integrated moving average (ARIMA) model and the 2-day predicted TEC product, named C2PG. While in the year with calm solar activity (2017), the prediction accuracy can be improved by $20.7 \%$ and $22.7 \%$.

\section{Introduction}

The ionosphere is an important part of the near-earth space, and it is distributed in the range of $60-1000 \mathrm{~km}$ from the Earth's surface [1]. Due to the influence of solar radiation and various cosmic rays, the atmospheric molecules in this part are ionized to a large number of free electrons. Total electron content (TEC) is an important parameter to characterize the degree of electromagnetic activity of the ionosphere, which, at a certain point, means the integral of free electrons on the propagation path of electromagnetic signals. The international unit is TECU $\left(1 \mathrm{TECU}=10^{16} \mathrm{el} / \mathrm{m}^{2}\right)$. Therefore, TEC is often used as an indicator to represent the ionospheric activity and the signal delay caused by the ionosphere.

In Global Navigation Satellite System (GNSS) applications, the delay encountered by satellite signals passing through the ionosphere is one of the main errors, which greatly restricts the performance of GNSS high-precision positioning [2-4]. Thus, effective prediction of TEC has always been very important for GNSS positioning and other related applications. For this problem, there are several commonly used methods including the International Reference Ionosphere model (IRI) [5], time series analysis [6], and artificial neural network $[7,8]$. The IRI models (e.g., IRI2007 and IRI2012) are empirical models proposed by the International Federation of Radio Science, based on longterm worldwide observations from ionosondes and radars and are combined with years of ionospheric research results. Although the model can obtain long-term ionospheric prediction results, the input parameters are more complicated and the error is large under certain conditions $[9,10]$. In 1999, Muhtarov and Kutiev [11] proposed an autocorrelation method for ionospheric interpolation and shortterm prediction. The autocorrelation or normalized autocorrelation parameters were determined based on past observation data, and the autocorrelation model was further obtained. This model can be used for temporal interpolation over a period of time and for extrapolation (prediction). Ratnam et al. [12] used the dual-frequency GNSS 
observations from the Indian low-latitude stations to make a short-term prediction of the vertical TEC according to the autoregressive moving average (ARMA) model. Compared with the original observation and the IRI2007 model, the ARMA model showed good performance during the magnetic storm period.

Because of the nonlinear characteristics of the ionospheric change, the artificial neural network is also widely used in the short-term prediction of the ionosphere $[13,14]$. Williscroft and Poole [15] firstly applied the neural network to the prediction of ionospheric foF2, which obtained better results than the IRI model. Based on error compensation, Zheng et al. [16] proposed an ionospheric foF2 prediction method, which combined the back propagation neural network (BPNN) and IRI2012 model. The prediction accuracy was improved by $19 \%$ compared with the IRI2012 model. The nonlinear autoregressive with exogenous input (NARX) is a kind of recurrent neural network (RNN) [17], which predicts future values from past values of the time series and second time series as inputs. It can establish the relationship between the two time series of inputs and outputs and has been demonstrated to be suitable for nonlinear time series modeling [18]. In addition, the NARX network has also been proven to perform better on issues involving long-term dependencies [19]. About the using of NARX on atmosphere research, Cai et al. [20] firstly used NARX to establish a model for predicting the SYM-H index using solar wind and interplanetary magnetic field (IMF) parameters in advance. The correlation coefficient was at least 0.9 even during a strong magnetic storm, which proved the practicability and performance of this method. It can be expected that NARX can also be used in the prediction of ionospheric TEC $[21,22]$. In this paper, a NARX-based ionospheric TEC prediction method is proposed. According to the previous TEC data and exogenous time parameters, the NARX is used for modeling for single-point TEC time series prediction and obtaining 2-day predicted TEC in advance.

\section{Materials and Methods}

2.1. NARX Neural Network. The inputs of NARX neural network contain not only the exogenous information of the current time but also the output data of the previous time, and thus the network has the feature of memory and feedback, making the information of system more complete. The mathematical expression of the NARX model can be expressed as follows:

$$
\hat{y}(t)=f\left(y(t-1), \ldots, y\left(t-n_{d}\right), x(t), \ldots, x\left(t-n_{d}\right)\right),
$$

where $\hat{y}(t)$ is the output value of the NARX at the time $t-1$ and the predicted value for the time $t . n_{d}$ is the number of input and output delays. $y(t-1), y(t-2), \ldots, y\left(t-n_{d}\right)$ are the past value of the time series. $x(t-1), x(t-2), \ldots, x(t-$ $n_{d}$ ) are the past value of the time series of exogenous inputs. $f$ is the nonlinear mapping function of the neural network, which is unknown and needs to be trained.

After feedback, the NARX network is similar in architecture to the multilayer perceptron (MLP), including the input layer, the hidden layer, and the output layer. Other parameters include the number of neuron nodes, the number of network layers, the connection weights, and the activation function. The MLP provides an effective nonlinear problem solution that can be used to train and obtain nonlinear mapping relationships.

The output of the hidden layer nodes $h_{i}(t)$ is

$$
h_{i}(t)=f_{A}\left[\sum_{j=1}^{n_{d}} \omega_{i j}(t) x(t-j)+\sum_{k=1}^{n_{d}} \omega_{i k}(t) y(t-j)+b_{i}\right] \text {. }
$$

where $f_{A}$ is the activation function of the hidden layer nodes. $\omega_{i j}(t)$ is the connection weights between the $i$ th hidden layer node and input neuron $x(t-j) . \omega_{i k}(t)$ is the connection weights between the $i$ th hidden layer node and feedback $y(t-k) . b_{i}$ is the bias of hidden layer nodes. The value of the output layer node $\hat{y}(t)$ is

$$
\hat{y}(t)=f_{A}\left[\sum_{i=1}^{N} \omega_{i}(t) h_{i}+b\right]
$$

where $\hat{y}(t)$ is the output value of the NARX network. $\omega_{i}(t)$ is the connection weight between the $i$ th hidden layer node and output layer. $b$ is the bias of the network. $N$ is the number of hidden layer nodes.

2.2. NARX for TEC Prediction. On the base of NARX, this paper chooses to use the International GNSS Service (IGS) TEC map as the ionospheric prediction target. After downloading the ionospheric TEC map files from IGS [23], the TEC at any point can be obtained by interpolation. Then, all TEC maps are interpolated for a period of time at the same point to constitute a single-point TEC time series. The time series $y$ can represent the change of TEC during this period, which can be expressed as follows:

$$
y=\left\{\mathrm{TEC}_{1}, \mathrm{TEC}_{2}, \ldots, \mathrm{TEC}_{i}\right\},
$$

where $i=1,2, \ldots, n_{d}$ is the length of time series.

The input parameters of NARX are the time factors related to the variations of TEC, which are selected based on the periodicity characteristics of ionosphere. Diurnal variation is considered as a distinct feature of TEC. The TEC increases to a maximum after noon and then decreases to a minimum during midnight. Season alternation is also an important factor. TEC is large in spring and autumn, but less in winter and summer, generally. So, the time information is taken as input parameters including local time (0-23), day of year (DOY, 1-365), and season. And then, these parameters are normalized by the sine and cosine with an interval $(-1,1)$. 


$$
x=\left[\sin \left(\frac{2 \pi}{24} h\right), \cos \left(\frac{2 \pi}{24} h\right), \sin \left(\frac{2 \pi}{365} D\right), \cos \left(\frac{2 \pi}{365} D\right), \sin \left(\frac{2 \pi}{4} S\right), \cos \left(\frac{2 \pi}{4} S\right)\right]^{T},
$$

where $h$ is the hour. $D$ is the DOY. $S$ is the virtual value expressing spring, summer, autumn, and winter with $1,2,3$, and 4. For different types of time data, the inputs are normalized to $[-1,1]$ using trigonometric functions with different periods. The length of the time delay is set to 2 days. When the sampling interval is $2 \mathrm{~h}, n_{d}$ is 24 . The number of hidden layer is 1 , the number of hidden layer nodes $N$ is 20 , and the activation function $f_{A}$ is selected as tanh. The structure of the NARX network is shown in Figure 1.

After training, the accuracy of prediction can be represented by root mean square error (RMSE), regression values (R), and relative error (REL). They can be expressed as follows:

$$
\begin{aligned}
\mathrm{RMSE} & =\sqrt{\frac{\sum\left(\mathrm{TEC}_{\text {pre }}-\mathrm{TEC}_{\mathrm{T}}\right)^{2}}{n}}, \\
R & =\frac{n \sum \mathrm{TEC}_{\mathrm{pre}} \mathrm{TEC}_{T}-\sum \mathrm{TEC}_{\mathrm{pre}} \sum \mathrm{TEC}_{T}}{\sqrt{n \sum \mathrm{TEC}_{\mathrm{pre}}^{2}-\left(\sum \mathrm{TEC}_{\mathrm{pre}}\right)^{2}}-\sqrt{n \sum \mathrm{TEC}_{T}^{2}-\left(\sum \mathrm{TEC}_{T}\right)^{2}}}, \\
\mathrm{Rel} & =\frac{1}{n} \sum\left|\frac{\mathrm{TEC}_{\mathrm{pre}}-\mathrm{TEC}_{T}}{\mathrm{TEC}_{T}}\right| \times 100 \% .
\end{aligned}
$$

where, $n$ is the number of samples. TEC $\mathrm{pre}_{\text {is }}$ is predicted TEC values. TEC ${ }_{T}$ is target TEC (IGS TEC maps are used as true values). RMSE shows the absolute error between the $\mathrm{TEC}_{\text {pre }}$ and $\mathrm{TEC}_{T}$. $R$ represents the correlation between the two sets of time series, and REL represents the relative error magnitude.

\section{Experiments and Results}

3.1. Data Preparation. The experimental TEC data comes from the global ionospheric TEC grid product (IONEX) released by IGS in 2011 and 2017, with an interval of 2 hours and a grid size of $2.5^{\circ} \times 5^{\circ}$. The three stations, different latitudes and basically the same longitude, in the northern hemisphere were selected to analyze the influence of latitude on the ionospheric prediction, namely, Beijing (BJ), Wuhan (WH), and Sanya (SY). The approximate coordinates of the stations are shown in Table 1. The TEC of the three stations in the zenith direction was interpolated according to the TEC map. The 1-year TEC series consists of daily average value which is shown in Figure 2.

In Figure 2, the TEC series have obvious seasonal changes. The TEC in spring and autumn are significantly higher than that in summer and winter, reflecting a trend of fluctuating, and the magnitude of the change is very large. By comparing the data of 2011 and 2017, it can be found that the TECs in 2011 are significantly higher than those in 2017 and even doubled in spring and autumn. In addition, due to the different latitudes of the three stations, it can be clearly seen that the TECs of the low-latitude area are higher than that of the high latitude area.

According to the activities of the ionosphere, the TEC data of four time periods are selected for TEC prediction experiments, and the relationship between ionospheric activity and prediction accuracy at each period are analyzed. The four datasets are called 2011-S, 2011-A, 2017-S, and 2017-A. A total of 1440 TEC values in each dataset are used as training samples in the previous 120 days, and 240 TEC data are used as test samples in the next 20 days. Three stations conduct the prediction experiments, for a total of 12 datasets. The datasets are shown in Table 2. Part of training samples is shown in Table 3.

According to the NARX ionospheric prediction algorithm proposed in this paper, the above 12 datasets are used for TEC prediction experiments. In experiments, the future TECs are predicted by the true values of 24 TEC values in the previous 2 days. Then, slide the predicted window forward until the completion of the 20-day prediction instead of one prediction for 20 days.

3.2. NARX Prediction. The NARX method is used to predict 20-day TECs using all datasets. The predicted TEC series in 2011 and 2017 are shown in Figures 3 and 4. The blue curve in the figures represents target TEC $\mathrm{TEC}_{T}$, and the red curve represents prediction TEC $\mathrm{TEC}_{\text {pre }}$. The error statistics and prediction performance are shown in Table 4.

Depending on the predicted RMSE of the different datasets, the periodicity of the ionospheric changes can also be proved. The trend of the predicted TEC and the target series are coincident, and the errors are in a small range. For the same period, the RMSE for predicted TEC of the three stations increases with the decreasing latitudes. Because the latitude of Beijing is highest, the prediction error is the smallest. On the contrary, the prediction error is the largest in Sanya. For different time periods, the RMSEs of the three stations are consistent with the ionospheric activity. When 


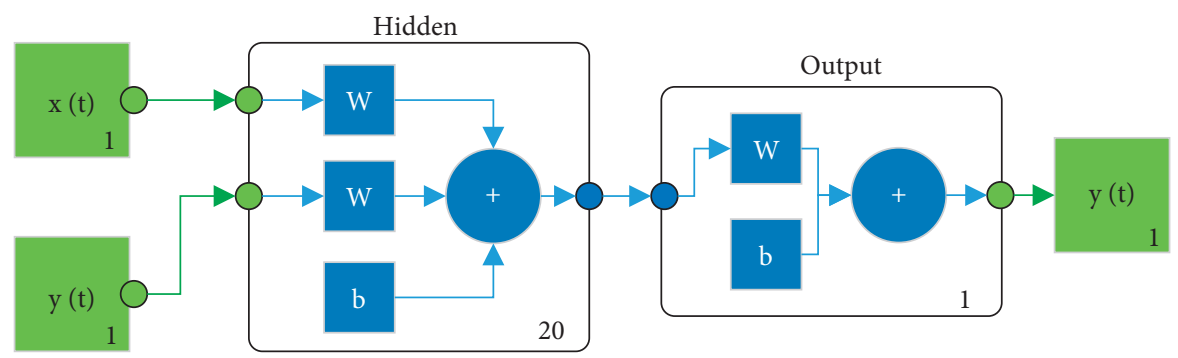

FIgURE 1: The structure of the NARX networks.

TABle 1: Station location.

\begin{tabular}{lcc}
\hline Station & Latitude & Longitude \\
\hline Beijing (BJ) & $40.3^{\circ}$ & $116.2^{\circ}$ \\
Wuhan (WH) & $30.5^{\circ}$ & $114.4^{\circ}$ \\
Sanya (SY) & $18.3^{\circ}$ & $109.6^{\circ}$ \\
\hline
\end{tabular}

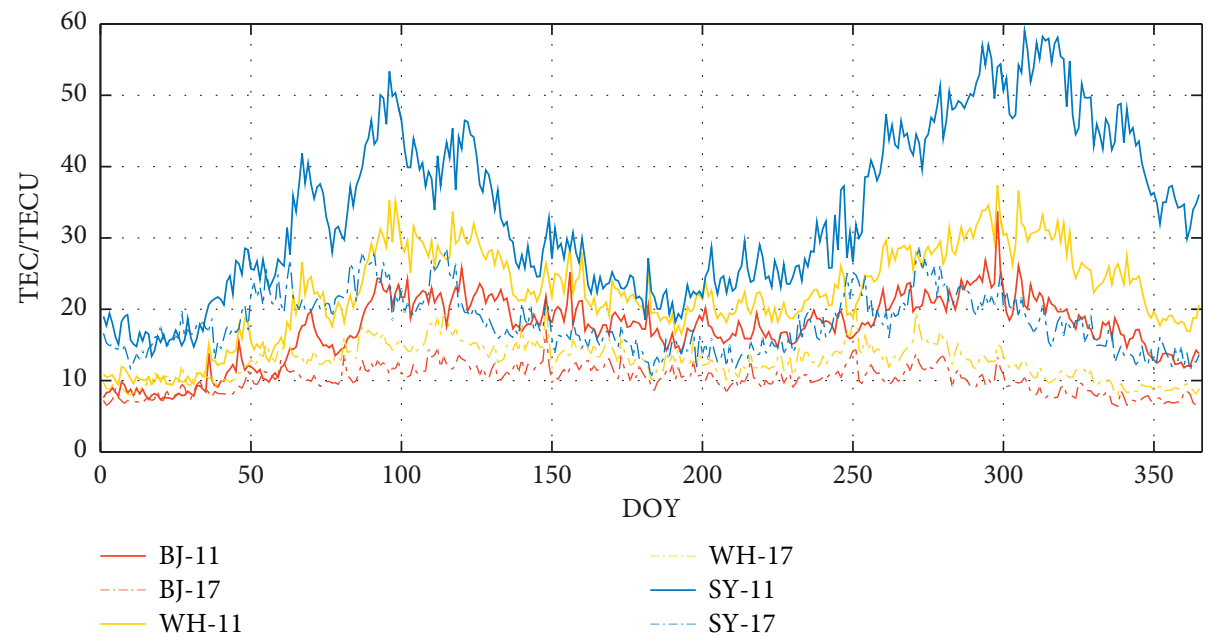

Figure 2: 1-year TEC series.

the ionosphere is active, the RMSEs are large, and the RMSEs are small when the ionosphere is calm. In general, the RMSE is the maximum in 2011-A, the minimum in 2017-S, and the RMSEs of 2011-S and 2017-A are roughly the same, which are between the first two. Overall, the minimum RMSE is 1.049 TECU in 2017-S-BJ, and the maximum RMSE is 3.722 TECU in 2011-A-SY.

However, the trends of RMSEs are not exactly the same for the relative errors. The RMSEs are related to the ionospheric activity and show periodic fluctuations with time of datasets, but RELs show an increasing trend with time, as shown in Figure 5. It is because the RELs are not only related to the prediction biases but also related to the magnitude of the target TECs as the denominators. Taking 2017-A as an example, although the prediction errors are small, the RELs are large due to the low level of TEC target value. In the 12 datasets, the RELs are less than $10 \%$ in 11 datasets, and the REL of only one dataset is $10.71 \%$. Therefore, we can explain that the relative error of NARX is better than $11 \%$.
In addition, the regression coefficients between the predicted values and the target values of the 12 datasets are all greater than 0.9 , indicating a very strong correlation.

3.3. Comparison with ARIMA and C2PG. We also used different prediction methods or products to compare with the NARX using the same datasets. Firstly, the 2-day forecast product released by CODE, namely, C2PG, is used for comparison, which can be downloaded on the IGS website in advance. It has the same format as IONEX. Secondly, the ARIMA method introduced by Box and Jenkins [24] is also adopted, and the main formula is as follows:

$$
y_{t}=\phi_{1} y_{t-1}+\phi_{2} y_{t-2}+\cdots \phi_{p} y_{t-p}+\varepsilon_{t}-\theta_{1} \varepsilon_{t-1}-\theta_{2} \varepsilon_{t-2}-\cdots \theta_{q} \varepsilon_{t-q} .
$$

The corresponding nonnegative integer $p$ is the autoregressive order, and $q$ is the moving average order. $\phi_{i}$ is the regression coefficient. $\theta_{i}$ is the moving average 
TABLE 2: Time setting of the TEC dataset.

\begin{tabular}{llr}
\hline Dataset & Train time period & Test time period \\
\hline $2011-\mathrm{S}$ & DOY 001-120, 2011 & DOY 121-140, 2011 \\
$2011-\mathrm{A}$ & DOY 141-260, 2011 2011 \\
$2017-\mathrm{S}$ & DOY 001-120, 2017 & DOY 261-280, 2017 \\
$2017-\mathrm{A}$ & DOY 141-260, 2017 & DOY 121-140, 2017 \\
\hline
\end{tabular}

Table 3: Part of training samples (0 h, DOY 121-14h, DOY 122, 2017).

\begin{tabular}{|c|c|c|c|c|c|c|c|c|}
\hline \multicolumn{3}{|c|}{ Target TEC } & \multicolumn{6}{|c|}{ Exogenous input } \\
\hline Beijing & Wuhan & Sanya & $\sin (2 \pi / 24 h)$ & $\cos (2 \pi / 24 h)$ & $\sin (2 \pi / 365 D)$ & $\cos (2 \pi / 365 D)$ & $\sin (2 \pi / 4 S)$ & $\cos (2 \pi / 4 S)$ \\
\hline 12.346 & 13.910 & 12.972 & 0.000 & 1.000 & 0.872 & -0.490 & 0.000 & -1.000 \\
\hline 14.001 & 16.231 & 20.362 & 0.500 & 0.866 & 0.872 & -0.490 & 0.000 & -1.000 \\
\hline 15.477 & 23.413 & 31.144 & 0.866 & 0.500 & 0.872 & -0.490 & 0.000 & -1.000 \\
\hline 16.130 & 23.898 & 35.085 & 1.000 & 0.000 & 0.872 & -0.490 & 0.000 & -1.000 \\
\hline 17.249 & 23.470 & 34.623 & 0.866 & -0.500 & 0.872 & -0.490 & 0.000 & -1.000 \\
\hline 16.358 & 19.306 & 32.900 & 0.500 & -0.866 & 0.872 & -0.490 & 0.000 & -1.000 \\
\hline 14.504 & 16.463 & 23.203 & 0.000 & -1.000 & 0.872 & -0.490 & 0.000 & -1.000 \\
\hline 11.869 & 11.228 & 11.454 & -0.500 & -0.866 & 0.872 & -0.490 & 0.000 & -1.000 \\
\hline 9.877 & 10.242 & 9.940 & -0.866 & -0.500 & 0.872 & -0.490 & 0.000 & -1.000 \\
\hline 9.347 & 10.005 & 9.173 & -1.000 & 0.000 & 0.872 & -0.490 & 0.000 & -1.000 \\
\hline 7.916 & 7.674 & 7.000 & -0.866 & 0.500 & 0.872 & -0.490 & 0.000 & -1.000 \\
\hline 9.117 & 8.993 & 6.388 & -0.500 & 0.866 & 0.872 & -0.490 & 0.000 & -1.000 \\
\hline 13.712 & 15.912 & 14.999 & 0.000 & 1.000 & 0.863 & -0.505 & 0.000 & -1.000 \\
\hline 15.501 & 18.637 & 21.218 & 0.500 & 0.866 & 0.863 & -0.505 & 0.000 & -1.000 \\
\hline 14.850 & 22.402 & 31.824 & 0.866 & 0.500 & 0.863 & -0.505 & 0.000 & -1.000 \\
\hline 15.093 & 26.151 & 38.289 & 1.000 & 0.000 & 0.863 & -0.505 & 0.000 & -1.000 \\
\hline 13.718 & 22.679 & 38.700 & 0.866 & -0.500 & 0.863 & -0.505 & 0.000 & -1.000 \\
\hline 14.000 & 20.388 & 34.468 & 0.500 & -0.866 & 0.863 & -0.505 & 0.000 & -1.000 \\
\hline 15.666 & 15.410 & 16.326 & 0.000 & -1.000 & 0.863 & -0.505 & 0.000 & -1.000 \\
\hline 11.298 & 9.284 & 9.457 & -0.500 & -0.866 & 0.863 & -0.505 & 0.000 & -1.000 \\
\hline
\end{tabular}

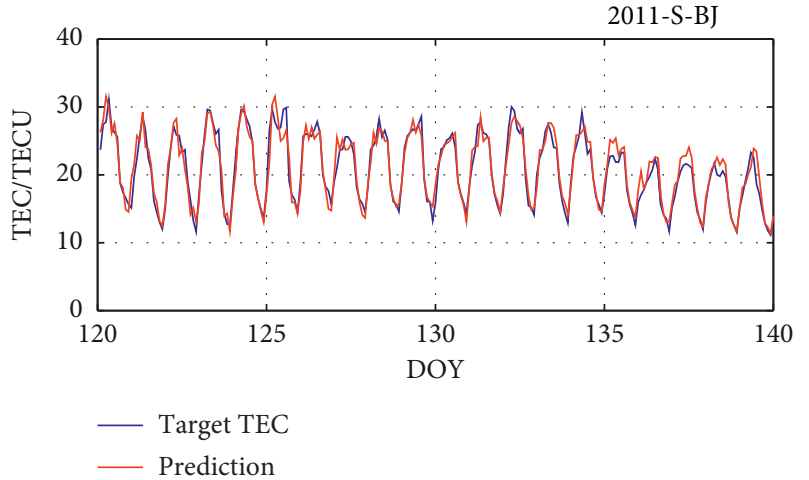

(a)

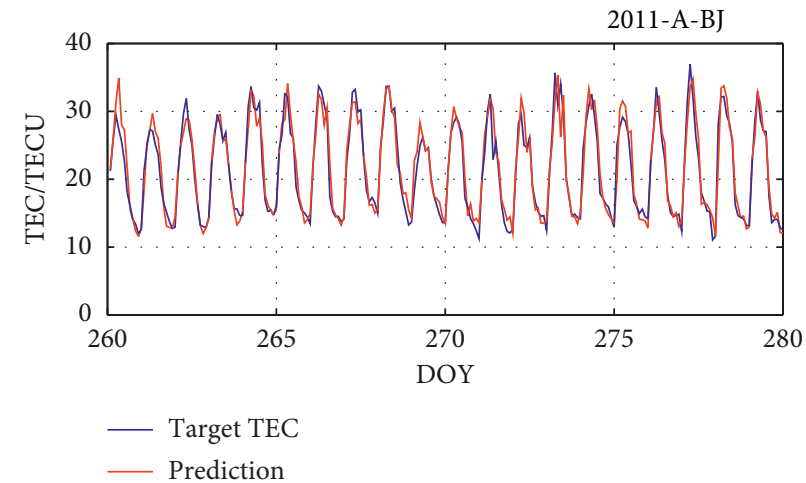

(b)

Figure 3: Continued. 


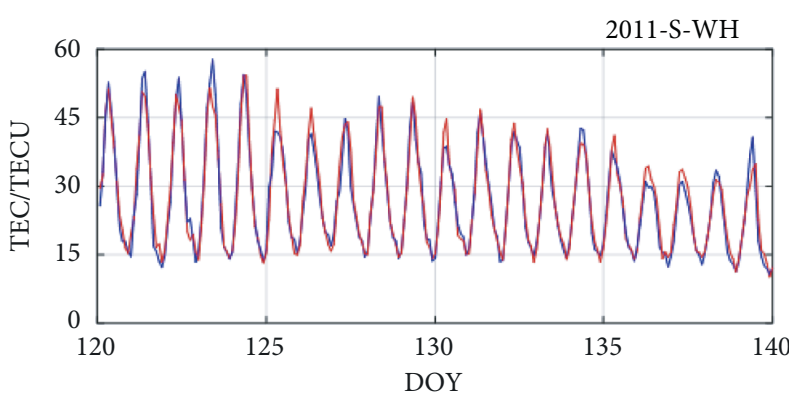

- Target TEC

— Prediction

(c)

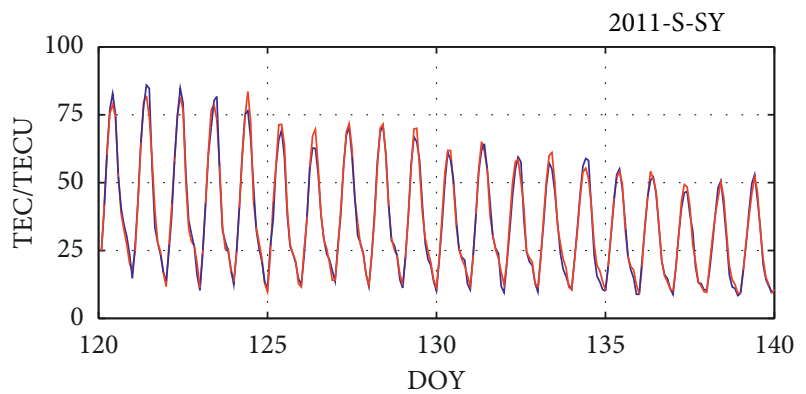

- Target TEC

_ Prediction

(e)

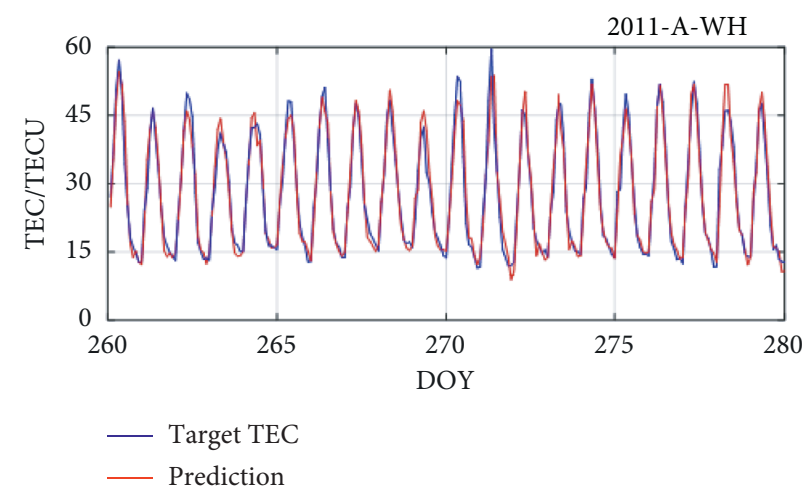

(d)

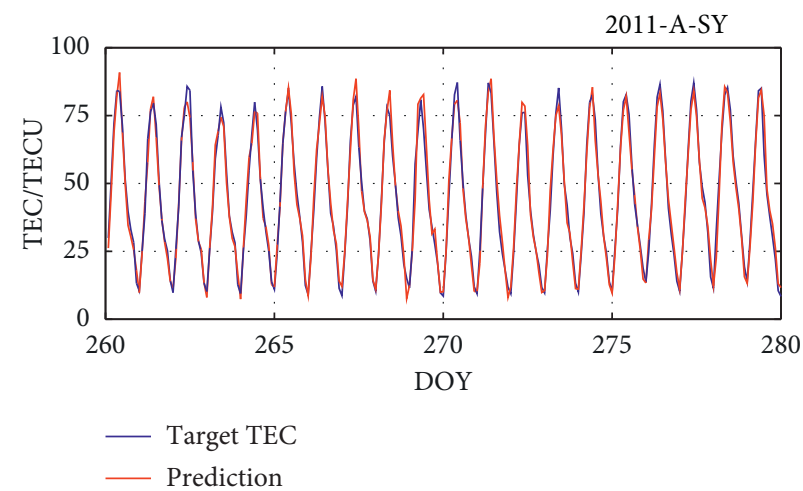

(f)

FIgURE 3: The predicted TEC and target TEC series in 2011.

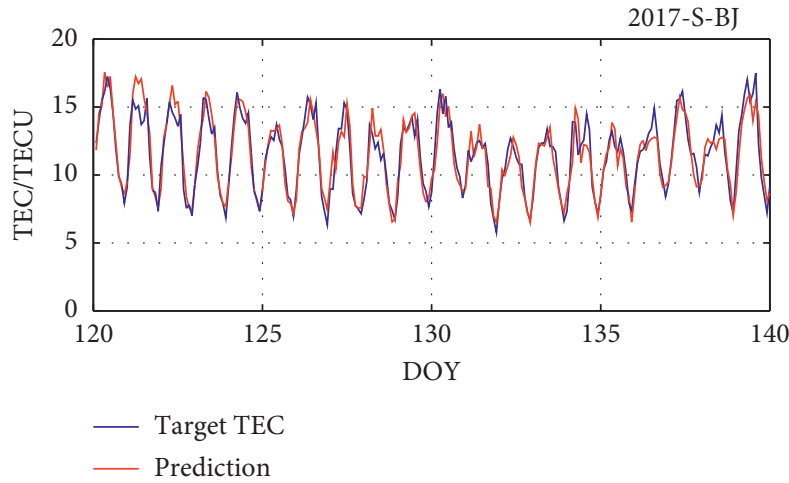

(a)

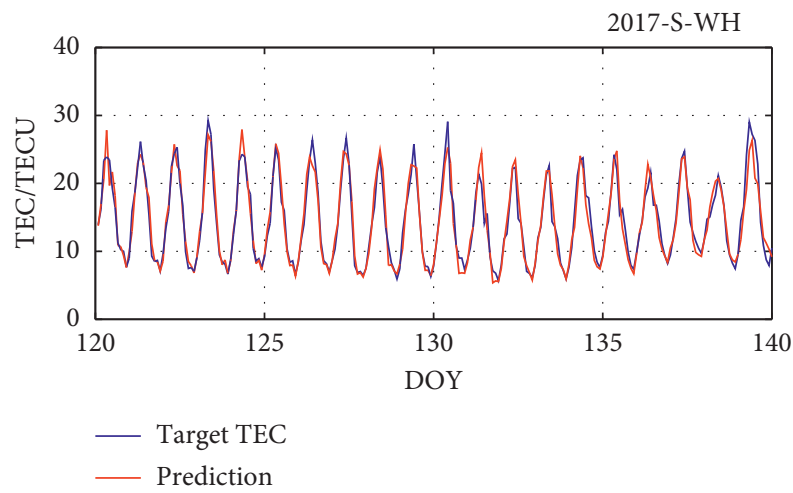

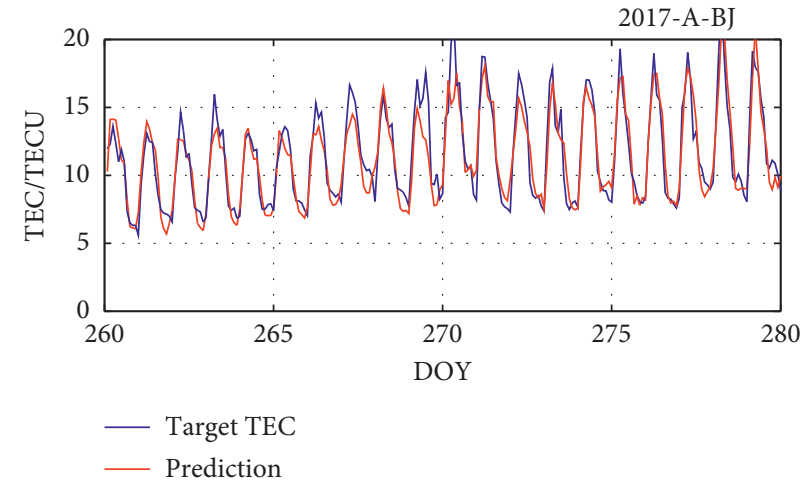

(b)

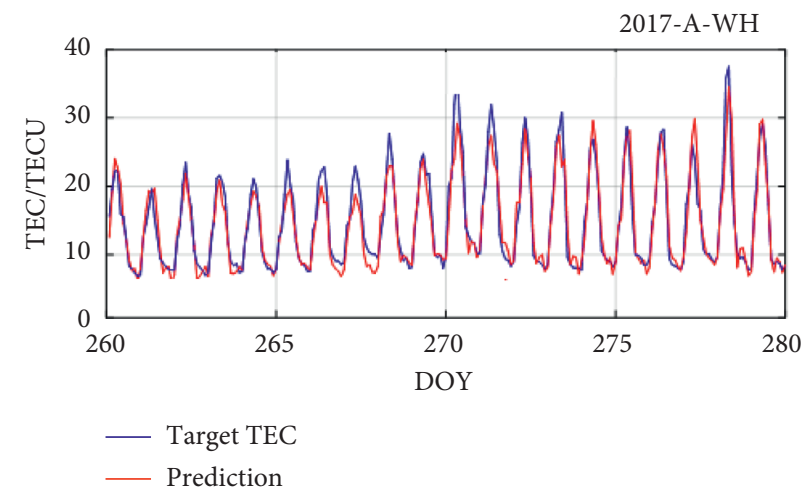

(d)

Figure 4: Continued. 


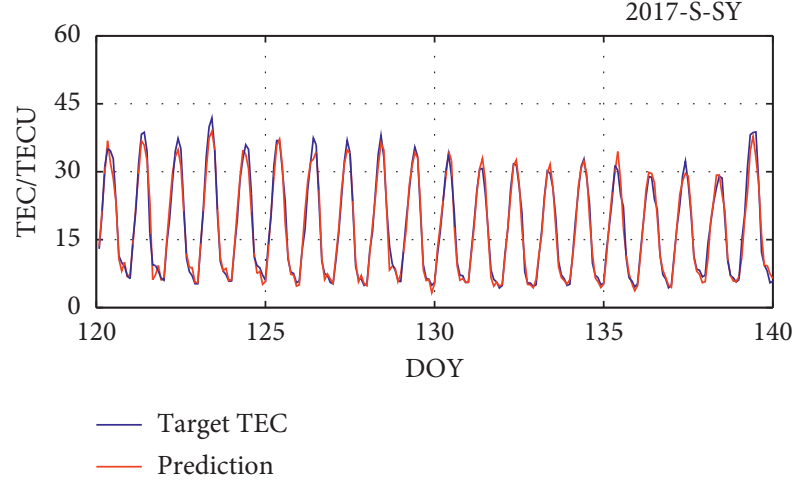

(e)

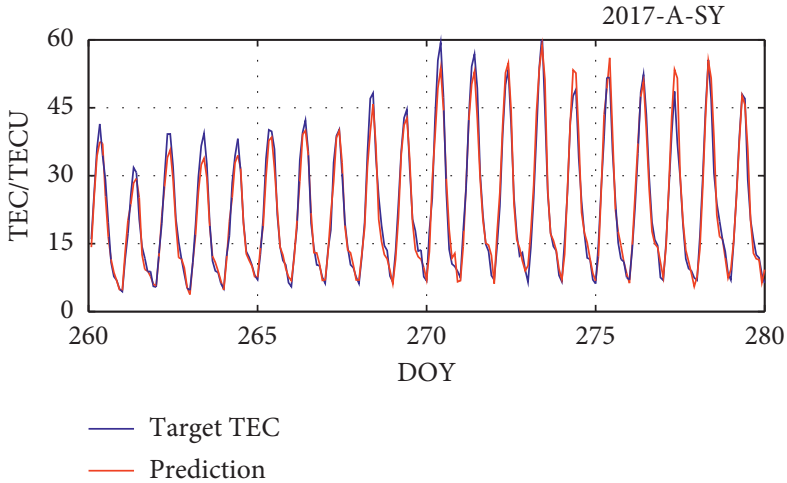

(f)

FIgure 4: The predicted TEC and target TEC series in 2017.

TABLE 4: The error statistic of the NARX.

\begin{tabular}{|c|c|c|c|c|}
\hline Time period & Station & RMSE/TECU & Regression & Relative error (\%) \\
\hline \multirow{3}{*}{ 2011-S } & Beijing & 1.501 & 0.956 & 5.34 \\
\hline & Wuhan & 2.501 & 0.975 & 7.43 \\
\hline & Sanya & 2.655 & 0.991 & 6.96 \\
\hline \multirow{3}{*}{ 2011-A } & Beijing & 1.990 & 0.959 & 6.79 \\
\hline & Wuhan & 2.742 & 0.977 & 7.50 \\
\hline & Sanya & 3.722 & 0.988 & 8.20 \\
\hline \multirow{3}{*}{ 2017-S } & Beijing & 1.049 & 0.924 & 6.96 \\
\hline & Wuhan & 1.642 & 0.964 & 8.11 \\
\hline & Sanya & 1.784 & 0.987 & 8.88 \\
\hline \multirow{3}{*}{ 2017-A } & Beijing & 1.362 & 0.925 & 9.24 \\
\hline & Wuhan & 2.068 & 0.957 & 9.34 \\
\hline & Sanya & 2.728 & 0.982 & 10.71 \\
\hline
\end{tabular}
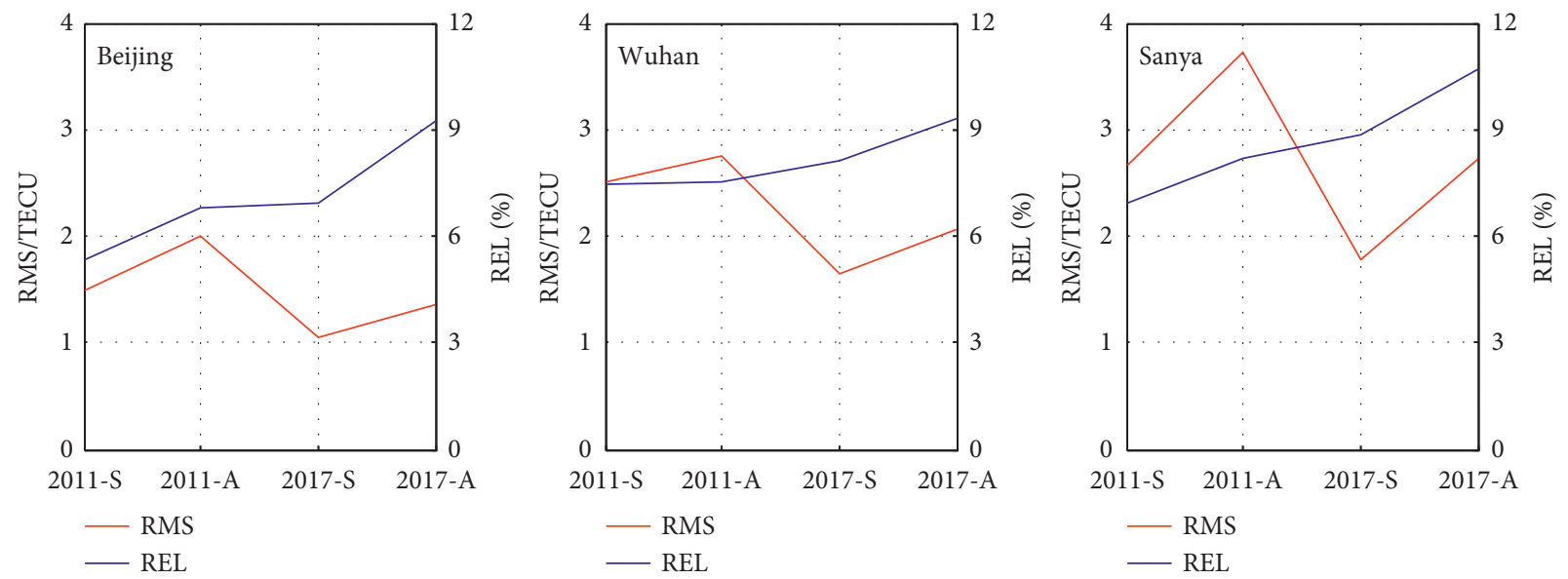

FIgURE 5: RMSEs and RELs in 12 datasets.

coefficient. $\varepsilon_{t}$ represents Gaussian white noise. In this article, after testing multiple dataset, the ARIMA model with $p=12$ and $q=4$ is adopted, and the data processing is the same as that of NARX. Taking the Wuhan station as an example, the residuals of the prediction results of the three methods are shown in Figure 6.
In Figure 6, the red line represents the prediction residuals of C2PG, the green line represents the ARIMA, and the blue line represents the NARX. Comparing the residuals in 2011 (Figures 6(a) and 6(b)) and 2017 (Figures 6(c) and 6(d)), the curves in 2011 fluctuate drastically than those in 2017, indicating that the 


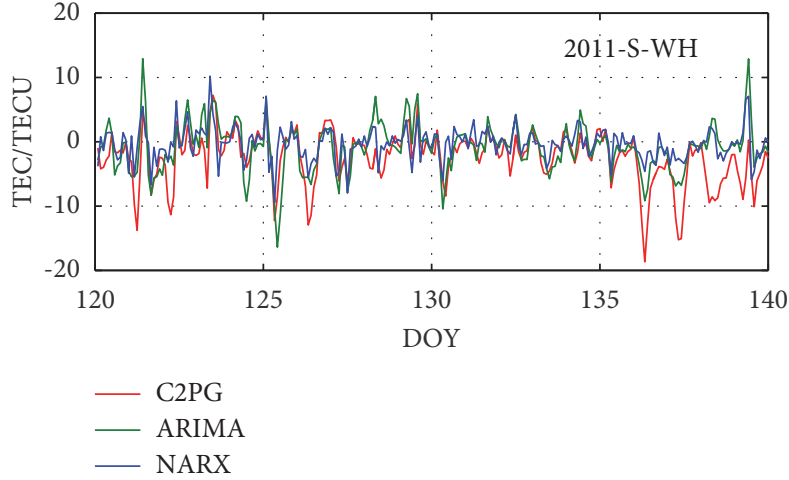

(a)

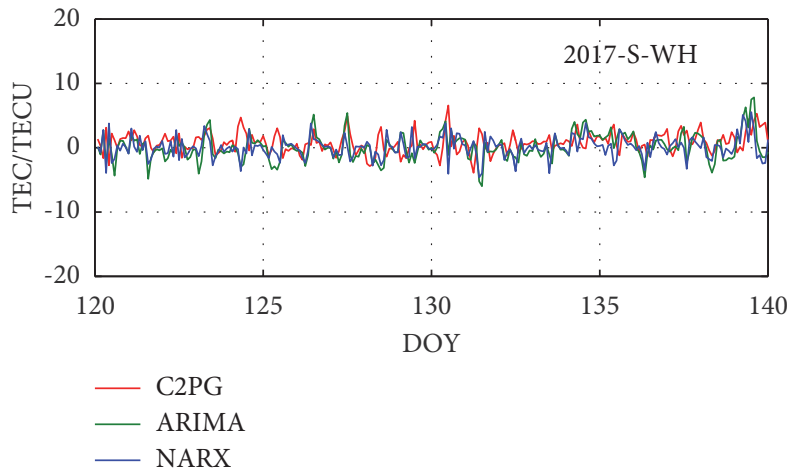

(c)

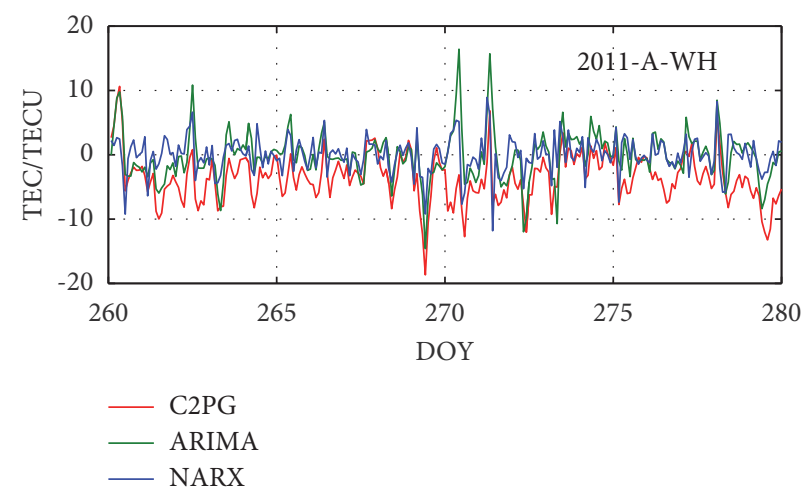

(b)

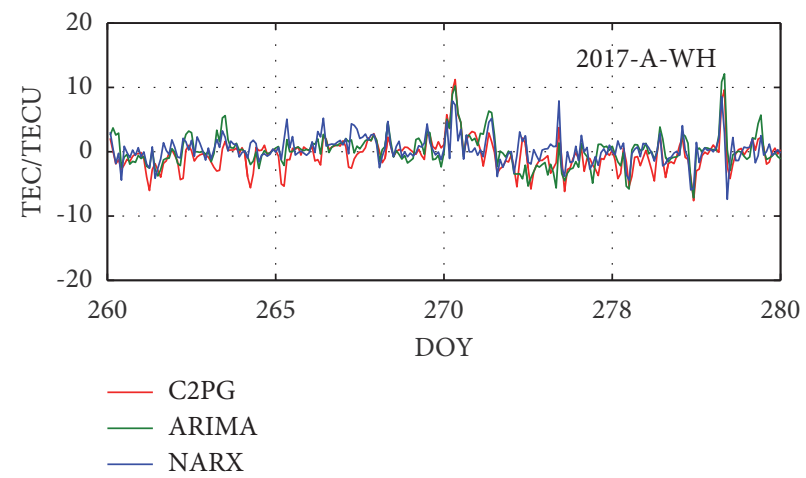

(d)

FIGURE 6: The residuals of the predictions with C2PG, ARIMA, and NARX in Wuhan.

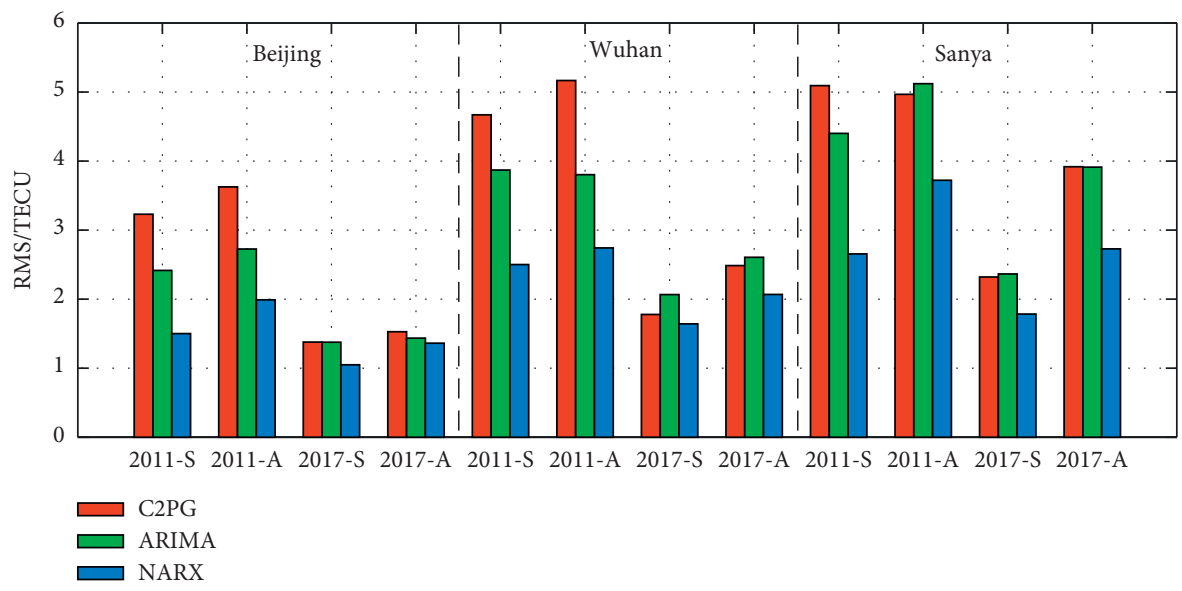

FIgURE 7: The RMSEs of the C2PG, the ARIMA, and the NARX in 12 datasets.

performances of 3 methods in the active period of the ionosphere are not as good as the calm period. Comparing the three prediction methods, the curve of NARX is more stable around the $x$-axis. There are more outliers in the residuals of the C2PG and ARIMA, and NARX has the better suppression of outliers. It is illustrated that the NARX has a good performance in TEC prediction in different periods and different areas.

The RMSEs of the three methods are shown in Figure 7 and Table 5. It can be seen that, during the active 
TABLE 5: The statistics of the three prediction methods (TECU).

\begin{tabular}{|c|c|c|c|c|}
\hline Dataset & Station & NARX & $\mathrm{C} 2 \mathrm{PG}$ & ARIMA \\
\hline \multirow{3}{*}{ 2011-S } & Beijing & 1.501 & 3.230 & 2.416 \\
\hline & Wuhan & 2.501 & 4.670 & 3.870 \\
\hline & Sanya & 2.655 & 5.093 & 4.401 \\
\hline \multirow{3}{*}{ 2011-A } & Beijing & 1.990 & 3.626 & 2.726 \\
\hline & Wuhan & 2.742 & 5.167 & 3.803 \\
\hline & Sanya & 3.722 & 4.967 & 5.121 \\
\hline \multirow{3}{*}{$2017-S$} & Beijing & 1.049 & 1.378 & 1.376 \\
\hline & Wuhan & 1.642 & 1.778 & 2.066 \\
\hline & Sanya & 1.784 & 2.321 & 2.365 \\
\hline \multirow{3}{*}{ 2017-A } & Beijing & 1.362 & 1.528 & 1.435 \\
\hline & Wuhan & 2.068 & 2.486 & 2.606 \\
\hline & Sanya & 2.728 & 3.918 & 3.912 \\
\hline \multirow{2}{*}{ Mean } & 2011 & 2.519 & 4.459 & 3.723 \\
\hline & 2017 & 1.772 & 2.235 & 2.293 \\
\hline
\end{tabular}

period of the ionosphere, the NARX has the greatest prediction accuracy, with an average of 2.519 TECU, followed by the ARIMA (3.723 TECU), and the C2PG (4.459 TECU) is the worst. In the ionospheric calm period, the accuracy of the NARX remains as high as 1.772 TECU. The accuracies of the C2PG and the ARIMA are equivalent, with 2.235 TECU and 2.293 TECU, respectively.

\section{Discussion and Conclusion}

In this paper, a TEC prediction method based on the NARX network is proposed. According to the periodic change of the ionosphere, the TEC information during a period of time is processed as a time series. The TEC series are treated as the inputs and outputs in the NARX at the meantime, and the time parameters are the exogenous inputs including hour, DOY, and season. In the experiments, the TEC data come from the IGS ionospheric grid products and can also use the actual raw observations of GNSS receiver. As a result, the experimental data and the time parameters are easy to obtain, and this method is easy to conduct in GNSS application.

According to the ionospheric activity, representative data in different periods are used for ionospheric TEC predictions. RMSE and REL are used to evaluate the prediction performance and compared with the IGS predicted products and the ARIMA method. The results show that the prediction using the NARX has the highest accuracy, and the RMSEs are between 1.501 TECU and 3.722 TECU in 2011 and between 1.049 TECU and 2.728 TECU in 2017. The accuracies of the ARIMA and C2PG are almost equivalent, and the mean RMSEs are 3.723 TECU and 4.459 TECU in 2011 and 2.293 TECU and 2.235 TECU in 2017. The NARX can have better prediction performance even during the active period of the ionosphere.

At the same time, the training of the NARX network requires a large amount of previous data, and the outputs are dependent on the training results heavily. So, the prediction results may not achieve satisfactory accuracy in the absence of past data or data without enough accuracy. And, we only use TEC map products in this paper, and the purpose is to verify the effectiveness and universality of the NARX algorithm in TEC prediction. Because the product is easy to obtain and has high accuracy, it can provide good experimental data.

For future works, we will further study the various characteristics of the ionospheric TEC and simplify or explore more input parameters related to the ionospheric TEC. The architecture of the NARX network will be optimized for improving the prediction accuracy of the ionospheric TEC. Raw GNSS observations will be used for TEC prediction with NARX network to improve the real-time performance and to weaken the system errors.

\section{Data Availability}

The global ionospheric products used in this paper are available at https://cddis.nasa.gov/archive/gnss/products/ ionex/(accessed on 1 October 2021).

\section{Conflicts of Interest}

The authors declare that they have no conflicts of interest.

\section{Acknowledgments}

This work was supported by the Fundamental Research Funds for the Central Universities (grant no. 2242020R40135) and the National Natural Science Foundation of China (grant nos. 41904022 and 41774027).

\section{References}

[1] L. Chen, W. Yi, and W. Song, "Evaluation of three ionospheric delay computation methods for ground-based GNSS receivers," GPS Solutions, vol. 22, no. 4, pp. 1-14, 2018.

[2] N. Wang, Z. Li, and Y. Yuan, "BeiDou Global Ionospheric delay correction Model (BDGIM): performance analysis during different levels of solar conditions," GPS Solutions, vol. 25 , no. 3, pp. 1-13, 2021.

[3] K. Su, S. Jin, and J. Jiang, "Ionospheric VTEC and satellite DCB estimated from single-frequency BDS observations with 
multi-layer mapping function," GPS Solutions, vol. 25, no. 2, pp. 1-17, 2021.

[4] Z. Li, N. Wang, L. Wang, A. Liu, H. Yuan, and K. Zhang, "Regional ionospheric TEC modeling based on a two-layer spherical harmonic approximation for real-time single-frequency PPP," Journal of Geodesy, vol. 93, no. 9, pp. 1659-1671, 2019.

[5] D. Bilitza, D. Altadill, V. Truhlik et al., "International Reference Ionosphere 2016: from ionospheric climate to realtime weather predictions," Space Weather, vol. 15, no. 2, pp. 418-429, 2017.

[6] C. Xie, Z. Mao, and G.-J. Wang, "Forecasting RMB exchange rate based on a nonlinear combination model of ARFIMA, SVM, and BPNN," Mathematical Problems in Engineering, vol. 2015, Article ID 635345, 10 pages, 2015.

[7] S. Tang, Z. Huang, and H. Yuan, "Improving regional ionospheric TEC mapping based on RBF interpolation," $A d$ vances in Space Research, vol. 67, no. 2, pp. 722-730, 2021.

[8] P. Xiong, D. Zhai, and C. Long, "Long short-term memory neural network for ionospheric total electron content forecasting over China," Space Weather, vol. 19, no. 4, Article ID e2020SW002706, 2021.

[9] L. M. Bjoland, V. Belyey, U. P. Løvhaug, and C. La Hoz, “An evaluation of International Reference Ionosphere electron density in the polar cap and cusp using EISCAT Svalbard radar measurements," Annales Geophysicae, vol. 34, no. 9, pp. 751-758, 2016.

[10] Z. Guo, Y. Yao, J. Kong et al., “Accuracy analysis of international reference ionosphere 2016 and NeQuick2 in the antarctic," Sensors, vol. 21, no. 4, p. 1551, 2021.

[11] P. Muhtarov and I. Kutiev, "Autocorrelation method for temporal interpolation and short-term prediction of ionospheric data," Radio Science, vol. 34, no. 2, pp. 459-464, 1999.

[12] D. V. Ratnam, M. Harshali, and M. V. G. Rao, "Forecasting of ionospheric time delays using ARMA model under Geomagnetic storm conditions," in Proceedings of the 2015 2nd International Conference on Signal Processing and Integrated Networks (SPIN), pp. 815-818, Noida, New Delhi, India, February 2015.

[13] W. Li, C. He, A. Hu, D. Zhao, Y. Shen, and K. Zhang, "A new method for improving the performance of an ionospheric model developed by multi-instrument measurements based on artificial neural network," Advances in Space Research, vol. 67, no. 1, pp. 20-34, 2021.

[14] K. A. Sidorenko and A. N. Kondratyev, "Improving the ionospheric model accuracy using artificial neural network," Journal of Atmospheric and Solar-Terrestrial Physics, vol. 211, Article ID 105453, 2020.

[15] L.-A. Williscroft and A. W. V. Poole, "Neural networks, foF2, sunspot number and magnetic activity," Geophysical Research Letters, vol. 23, no. 24, pp. 3659-3662, 1996.

[16] D. Zheng, W. Hu, and P. Li, "Predicting ionospheric critical frequency of the F2 layer over Lycksele using the neural network improved by error compensation technology," Survey Review, vol. 48, no. 347, pp. 130-139, 2016.

[17] E. Rangel, E. Cadenas, R. Campos-Amezcua, and J. L. Tena, "Enhanced prediction of solar radiation using NARX models with corrected input vectors," Energies, vol. 13, no. 10, p. 2576, 2020.

[18] Y. Xiu and W. Zhang, "Multivariate chaotic time series prediction based on NARX neural networks[C]," in Proceedings of the 2017 2nd International Conference on Electrical, Automation and Mechanical Engineering, Guangzhou, China, September 2017.
[19] Y. Bengio, P. Simard, and P. Frasconi, "Learning long-term dependencies with gradient descent is difficult," IEEE Transactions on Neural Networks, vol. 5, no. 2, pp. 157-166, 1994.

[20] L. Cai, S. Y. Ma, and Y. L. Zhou, "Prediction of SYM-H index during large storms by NARX neural network from IMF and solar wind data," Annales Geophysicae, vol. 28, no. 2, pp. 381-393, 2010.

[21] S. Kalita, B. Chetia, and M. Devi, "Prediction of total electron content using nonlinear autoregressive models with exogenous input recurrent neural network," Global Journal of Computer Science and Technology, 2017.

[22] C. Cesaroni, L. Spogli, A. Aragon-Angel et al., "Neural network based model for global Total Electron Content forecasting," Journal of Space Weather and Space Climate, vol. 10, p. 11, 2020.

[23] Z. Li, N. Wang, and M. Hernández-Pajares, "IGS real-time service for global ionospheric total electron content modeling," Journal of Geodesy, vol. 94, no. 3, pp. 1-16, 2020.

[24] M. Geurts, G. E. P. Box, and G. M. Jenkins, "Time series analysis: forecasting and control," Journal of Marketing Research, vol. 14, no. 2, p. 269, 1977. 